\title{
Induction of an illusory shadow person
}

\section{Stimulation of a site on the brain's left hemisphere prompts the creepy feeling that somebody is close by.}

The strange sensation that somebody is nearby when no one is actually present has been described by psychiatric and neurological patients, as well as by healthy subjects, but it is not understood how the illusion is triggered by the brain ${ }^{1,2}$. Here we describe the repeated induction of this sensation in a patient who was undergoing presurgical evaluation for epilepsy treatment, as a result of focal electrical stimulation of the left temporoparietal junction: the illusory person closely 'shadowed' changes in the patient's body position and posture. These perceptions may have been due to a disturbance in the multisensory processing of body and self at the temporoparietal junction.

The patient was a 22-year-old woman of normal psychiatric history who was undergoing evaluation for surgical treatment of epilepsy (see supplementary information). We identified an area on the left temporoparietal junction in her brain (Fig. 1a) where focal electrical stimulation repeatedly produced a feeling of the presence of another person in her extrapersonal space.

When stimulated at this region (for methods, see supplementary information; current amplitude, $10.0 \mathrm{~mA}$ ) in a supine position, the patient had the impression that somebody was behind her. Further stimulation $(10.0-11.0 \mathrm{~mA} ; n=3)$ induced the same experience, with the patient describing the "person" as young and of indeterminate sex, a "shadow" who did not speak or move, and whose position beneath her back was identical to her own ("He is behind me, almost at mybody, but I do not feel it" ; Fig. 1b).

During the next stimulation (11.0 mA; $n=1)$, the patient sat and embraced her knees with her arms (Fig. 1c). She noted that the "man" was now also sitting and that he was clasping her in his arms, which she described as an unpleasant feeling. Further stimulations (11.0 mA; $n=2$ ) were applied while the seated patient performed a naming (language-testing) task using a card held in her right hand (Fig. 1d): she again reported the presence of the sitting " son", this time displaced behind her to her right and attempting to interfere with the execution of her task ("He wants to take the card"; " $\mathrm{He}$ doesn't want me to read ${ }^{\text {}}$ ). Similar effects were observed for different positions and postures (see supplementary information) when stimuli exceeding $10 \mathrm{~mA}$ were applied to the same site on the left temporoparietal junction.

The sensation of a presence, as reported by this patient, has been described in people

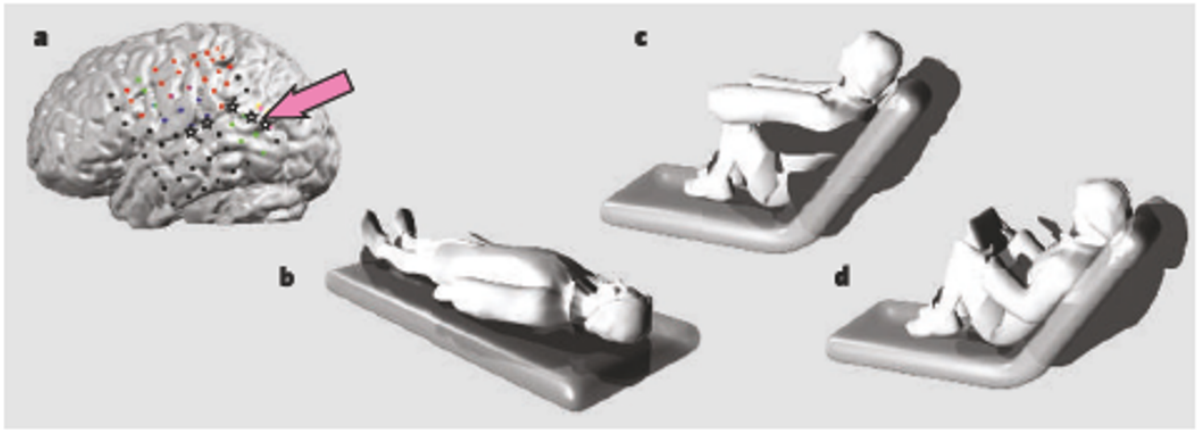

Figure 1| Me and my shadow. a, Three-dimensional surface reconstruction of the left hemisphere of the brain from magnetic resonance imaging. Subdural electrodes were implanted in the brain of an epileptic patient undergoing presurgical evaluation ${ }^{10}$. The locations at which focal electrical stimulation evoked different responses are shown (red, motor; blue, somatosensory; green, language; pink, site where 'feeling of a presence' could be induced (arrow)); stars indicate the epileptic focus. Since undergoing a left-temporal lobectomy, the patient has been free of seizures. b-d, Drawings showing relative positions and postures of the patient's body (white) and that of the illusory person (shaded) during cortical stimulation (illustrations, M. Boyer).

with psychiatric and neurological disorders ${ }^{1-4}$. Because it was possible to induce this feeling repeatedly from the temporoparietal junction, and because the illusory person closely mimicked the patient's body posture and position, we conclude that the patient was experiencing a perception of her own body ${ }^{1,4}$.

The temporoparietal junction is known to be involved in self-processing, self-other distinction, the integration of multisensory body-related information, and other illusory own-body perceptions ${ }^{5,6}$. We therefore propose that electrical stimulation of this area in our patient disturbed multisensory (proprioceptive and tactile) and sensorimotor integration of information with respect to her body, leading to the appearance of a first-rank schneiderian symptom of schizophrenia in a person with no psychiatric history. It is notable that hyperactivity in the temporoparietal cortex of patients with schizophrenia may lead to the misattribution of their own actions to other people ${ }^{7}$.

In conclusion, we argue that multisensory and/or sensorimotor disintegration ${ }^{s}$ at the temporoparietal junction due to electrical stimulation may lead to an own-body illusion of another person in near extrapersonal space. Although our patient was aware of the similarity between her own postural and positional features and those of the illusory person, she did not recognize that that person was an illusion of her own body, like many deluded schizophrenic patients ${ }^{389}$. Our findings may be a step towards understanding the mechanisms behind psychiatric manifestations such as paranoia, persecution and alien control. They also highlight the importance of multimodal mechanisms at the temporoparietal junction in human self-attribution.

Shahar Arzy ${ }^{\star} \neq$, Margitta Seeck $\neq \neq$, Stephanie Ortigue $\$ s$, Laurent Spinellit, Olaf Blanke ${ }^{\star}$ *Laboratory of Cognitive Neuroscience, Ecole Polytechnique Fédérale de Lausanne, Lausanne 1015, Switzerland e-mait: olaf.blanke@epfl.ch

†Presurgical Epilepsy Evaluation Unit, †Department of Neurology, University Hospital, Geneva 1211, Switzerland

§Center for Cognitive Neuroscience, Dartmouth

College, Dartmouth, New Hampshire 03755, USA

1. Bruggec, $P_{7}$ Regard, M.\& Landis, T. Neuropsychiat. Neurassychal. Behav. Neural. 9,114-122(1996).

2 Critchley, M.Acta Aychibt. Neural. Scand 30, 155-168 (1955).

3 Jaspers, K General Aychopathalogy (Manchester Univ. Press Manchester, 1972).

4. Blanke, $Q$, Ortigue, $S$, Coestaux, A, Martory, M.D. \& Landis, T.Neurocose 9, 329-339 (2003).

5. Blanke, $O$, Landis, T, Spinelli, L.\& Seeck, M. Brain 127, 243-258 (2004).

6. Ruby, P.\& Decety, 1. Nature Neurosci. 4, 546-550 (2001)

7. Farrer, C.etal. Aychibtr. Res 131,31-44 (2004).

8. Blakemore, S. -L, Wolpert, D. M.\& Frith, C. D. Trends Cogn Sci. 6, 237-242(2002).

9. Thompson, C. B. J. Psychiat. 141, 628-630 (1982).

10. Blanke, $O$, Perrig S., Thut, G, Landis, T.\& Seeck, M. J. Neurol. Neurosurg. Ayshiatr. 69,553-556 (2000).

Supplementary information accompanies this communication on Nature's website.

Received 19 July; accepted 25 A ugust 2006.

Competing financial interests: declared none. doi: $10.1038 / 443287 a$

BRIEF COMMUNICATIONS ARISING online - www.nature.com/bca see Nature contents. 